\title{
Beam Energy Considerations for Gold Nano-Particle Enhanced Radiation Treatment
}

\author{
F. Van den Heuvel Ph.D. ${ }^{* 1}$, Jean-Pierre Locquet Ph.D. ${ }^{2}$ \\ and S. Nuyts M.D. Ph.D. ${ }^{1}$ \\ Dept of Experimental Radiotherapy University of Leuven, Leuven, Belgium \\ E-mail: $\left({ }^{*}\right)$ frank. vandenheuvel@med.kuleuven.be \\ Solid State Physics and Magnetism Section University of Leuven, Leuven, \\ Belgium
}

\begin{abstract}
Purpose: A novel approach using nano technology enhanced radiation modalities is investigated. The proposed methodology uses antibodies labeled with organically inert metals with a high atomic number. Irradiation using photons with energies in the kilo-electron volt $(\mathrm{keV})$ range show an increase in dose due to a combination of an increase in photo-electric interactions and a pronounced generation of Auger and/or Coster-Krönig (A-CK) electrons.

Methods: The dependency of the dose deposition on various factors is investigated using Monte Carlo simulation models. The factors investigated include: agent concentration, spectral dependency looking at mono-energetic sources as well as classical bremsstrahlung sources. The optimization of the energy spectrum is performed in terms of physical dose enhancement as well as the dose deposited by Auger and/or Coster-Krönig electrons and their biological effectiveness.

Results: A quasi-linear dependency on concentration and an exponential decrease within the target medium is observed. The maximal dose enhancement is dependent on the position of the target in the beam. Apart from irradiation with low photon energies $(10-20 \mathrm{keV})$ there is no added benefit from the increase in generation of Auger electrons. Interestingly, a regular $110 \mathrm{kVp}$ bremsstrahlung spectrum shows a comparable enhancement in comparison with the optimized mono-energetic sources.

Conclusions: In conclusion we find that the use of nano-particle enhanced shows promise to be implemented quite easily in regular clinic on a physical level due to the advantageous properties in classical beams.
\end{abstract}




\section{Introduction}

Recently, methodologies using monoclonal antibodies that target specific tumor cells have been used to bring active compounds in the vicinity of these cells. One approach uses radioactive compounds of $\alpha$ - or $\beta$-emitters[1, 2, 3]. Alternatively, chemotherapeutic compounds have been attached to this delivery mechanism. The use of such approaches is interesting but limited due to the fact that the therapeutic compound is already active at time of delivery and during secretion by the body. More in particular with radioactive compounds an important whole body dose (red marrow dose) as well as renal toxicity are limiting factors for the efficacy of the treatment $[4,5,6,7]$.

It is the goal of this paper to investigate a delivery method that could potentially have most of the benefits associated with the previously listed therapeutic modalities ánd has almost none of the disadvantages. Which means:

(i) Differentiation between malignant and healthy cells.

(ii) Enhanced effectiveness.

(iii) Image guidance possibilities.

(iv) Activation methodology (i.e. Only active were it needs to be active).

(v) Large therapeutic window.

Dose enhancement due to the presence of gold nano-particles has been proposed already both by means of an injectable contrast agent as by the use of mono-clonal antibodies or other targetted delivery methods.. However, the enhancement relied on an increased interaction due to the increased probability of the photo-electric interaction being dependent on the atomic number at the proposed energies and the specific contribution of Auger electrons was not investigated[8, 9, 10, 11, 12]. All sources used in these studies were spectral sources and/or brachytherapy sources. Moreover, proposals to use more sophisticated photon sources have been put forward, in the hope to maximize the efficiency of the conversion of the beam energy to deposited energy as well as generate a high amount of Auger electrons[13].

In the dose deposition model proposed here a significant part of the energy is deposited by Auger electrons. There is reason to believe that Auger electrons deposit their energy more efficiently than those emanating from Compton or photo-electric effect processes. The exact mechanism behind this apparant dose enhancement effect is still unclear. A possible cause is the fact that Auger have a very low energy and deposit all of the energy within a range comparable to a few cell diameters. Furthermore, there is a possible change in the stopping power energy dependency at very low energies $(<10 \mathrm{keV})$, where the Bethe formalism breaks down. Alternatively, it could be that on average more than a single Auger electron is being produced, increasing the probability of clustered double strand breaks.

A number of authors have investigated the biological effects indirectly and support the notion that Auger electrons indeed have high LET characteristics[14, 15, 16].

To our knowledge. a systematic study of the impact of different spectral sources on the enhancement and the possible biological enhancement has not been published.

\section{Methods and Materials}

To perform the planning simulation we used MCNPX (Monte Carlo N-Particle eXtended)[17] version 2.7a running on a 738 node cluster at the University of Leuven. 
The department of experimental radiotherapy at Leuven is part of the beta-test group for MCNPX. The following physical parameters were used during these simulations.

Photon energy cutoff: $1 \mathrm{keV}$.

electron energy cutoff: $1 \mathrm{keV}$.

EM interaction library: ENDF/B-VI Release 8 Photoatomic Data 02/07/03

For the spectral dose deposition from Auger electrons version 2.7b was used. As the version is only available to use as a single processor binary it was not possible to run this on the cluster. Therefore if we did not need information on the Auger electrons seperately we chose to use the earlier version.

\subsection{Geometry}

The simulation geometry consisted of a tank filled with water containing three $1 \mathrm{~mm}$ thick cylindrical slabs holding tissue as defined by ORNL report TM8381. One slab was positioned at the surface of the tank representing a skin surface. A second slab was positioned at $5 \mathrm{~cm}$ distance from the tank surface down stream from the source. In this slab varying concentrations of gold were added in a homogeneous distribution. Additionally, geometrically identical slabs containing tissue, were positioned downstream adjacent to the structure containing the gold particles. The source in this geometry was a plane source (not divergent). The divergence can be introduced depending of the position of the source in a clinical situation by applying a inverse square rule.

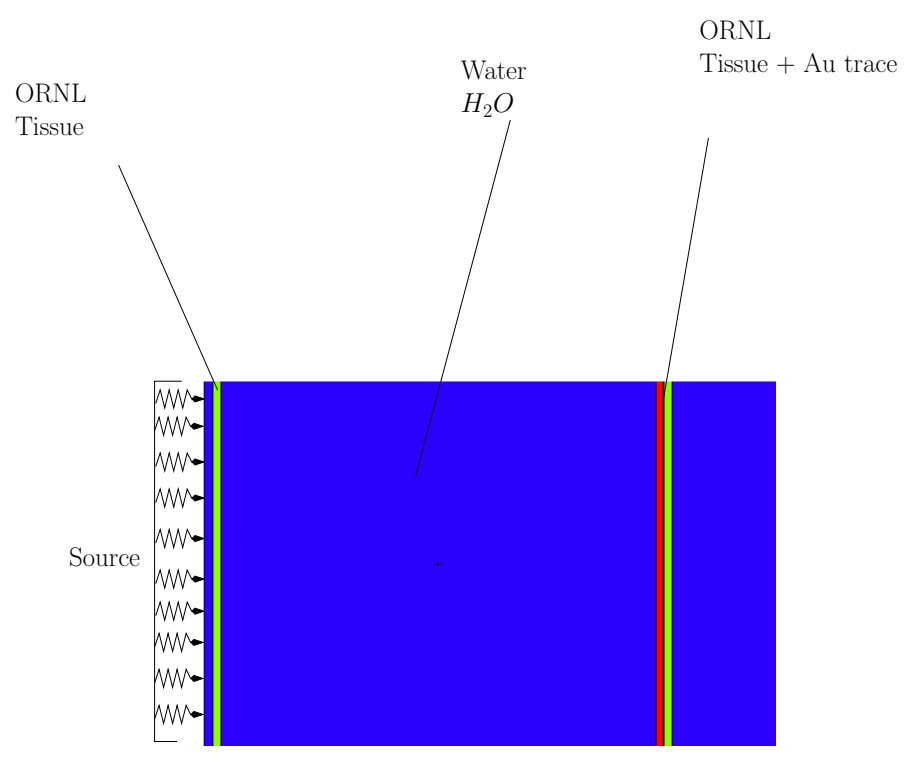

Figure 1: The geometry used in this simulation. The geometry has three volumes where dose can be measured: A skin section at the edge of the area, a target volume, and an organ at risk volume adjacent to the target volume. Alternatively the third volume can be used as a layer of target volume to study the effect of upstream nano-particles. 


\subsection{Source}

The radiation source is modeled after mono-energetic radiation sources obtained by Bragg-Gray diffraction of regular X-ray sources. Although these sources are called mono-energetic, they do exhibit some spectral spread which was modeled as a normal distribution with a $\sigma=1.5 \mathrm{keV}$.

\subsection{Simulations}

2.3.1. Energies To investigate the energy dependence of the therapeutic window, we performed the same simulation with quasi-mono-energetic beams of energies ranging from 10 to $200 \mathrm{keV}$, with special consideration to the $\mathrm{K}_{\alpha}$-energy of gold (i.e. $80.67 \mathrm{keV}$ ). Additionally, a broad spectrum beam was investigated. The spectrum was taken to be identical to that coming from an Acuity simulator's X-ray tube (Varian Inc.) running at $110 \mathrm{kVp}$.

2.3.2. Concentrations The medium used in the activated environment was considered to be tissue as defined in the (Oak Ridge National Labs (ORNL) Report TM-8381). Gold $(\mathrm{Z}=79)$ in natural isotope abundance was added with all other components diminished to yield a normalized weight. The structures were embedded in water. The concentrations of gold in one of the structures varied between 0 and $10 \%$ in steps of $1 \%$. A concentration of $10 \%$ is highly unlikely, however as reported by Verhaegen et al. it is the concentration of off-the-shelf contrast material and should serve as an upper limit of the enhancements achievable with this technique. Furthermore, it is to be expected that once a distribution methodology for the gold particles is implemented we are bound to see very heterogeneous concentrations of gold in the irradiated medium, reaching high concentrations locally.

All concentration related simulations were performed with the broad spectrum, for reasons made clear in the results section.

2.3.3. Auger electrons As mentioned above, the contribution of the Auger electrons could be estimated by tagging the electrons released due to Auger cascades in the cell of interest. The number of generated Auger electrons could then be linked to the energy of the source.

This to find the optimal energy for Auger electron generation. Below energies of $1 \mathrm{keV}$ MCNPX does not track the electrons and the energy is considered to be deposited locally. The simulations were performed for all energies as listed above.

\subsection{Analysis}

2.4.1. Energy Dependence The energy dependence is reviewed for the maximal concentration of $10 \%$, this because any differences between the energies would be magnified, as well as reduce the statistical errors in our monte carlo calculations. We define three types of dose enhancement:

Absolute Enhancement $\left(E_{a}\right)$ : The ratio of the dose deposited in the goldcontaining structure to the dose deposition in a run with exact same geometry without gold present. 
OAR Enhancement $\left(E_{O A R}\right)$ : The ratio of the dose deposited in the goldcontaining structure to the dose deposited in another structure in the geometry not containing gold, representing an Organ at Risk (OAR).

Skin ratio $\left(E_{S}\right)$ : The ratio of the dose deposited in the gold-containing structure to a layer $1 \mathrm{~mm}$ under the skin. Which is a special case of $E_{O A R}$.

We determined the energy deposited per unit mass by counting the energy deposited by electrons using the MCNPX tally F6:E. This underestimates the effectively deposited dose slightly as it does not take into account the energy expended to generate the photon initiated ionization. However, this ionization is likely not contributing to a biological effect as it is mainly through interaction with the gold atom which does not form part of the cell structure. The difference between F6:E and F6:P (Energy transferred by photons, which includes electrons) is about $1 \%$. However the electron energy deposition is better defined spatially.

2.4.2. Concentration The results from the concentration study were analyzed in function of absolute enhancement. The variation $E_{a}$ was fit using a linear relationship and a second order polynomial. The fit was performed using a minimization of a $\chi^{2}$-function taking into account the simulation errors.

\subsection{Radiobiological effect}

To estimate the relative effect of the change in spectrum and the increase in the contribution by Auger-electrons we used a fast monte carlo model of biological damage as proposed by Semenenko and Stewart[18] which has been shown to obtain the same results as track code monte carlo codes as proposed by Nikjoo et al. [19]. The approach used here determines the amount of different damage to a DNA-molecule from direct ionization as well as through the generation of radicals. It then produces a yield $(y)$ in percentage of the different types of damage ranging from single strand breaks to complex clustered double strand breaks for interacting electrons of a given energy $(E)$. The methodology used to incorporate this information in our calculations is as follows.

(i) For every energy available in the energy deposition histogram we used the code provided by Semenenko and Stewart to generate the yields of the different types of DNA-damage making sure that the lowest energy is included. The yield is given as percentage per cell and per Gy $\left(\% \mathrm{cell}^{-1} \mathrm{~Gy}^{-1}\right)$.

(ii) The data was fit as a function of energy of the electron using an equation of the form:

$$
y(E)=a+b E^{c}
$$

with $a, b$, and $c$ variable parameters. Figure 2 shows $y(E)$ for single strand breaks and double strand breaks together with the fitted parameters, which are provided in table 1.

(iii) The total relative yield of damage of type $\mathrm{D}\left(Y_{D}\right)$ was then given by

$$
Y_{D}=\sum_{E=E_{\min }}^{E_{\max }} y_{D}(E) F(E)
$$

With $\mathrm{F}(\mathrm{E})$ the normalized histogram of deposited energy as a function of energy of the depositing electrons (in MCNPX tally F6:E divided by the total energy). 


\begin{tabular}{c|c|c|} 
par & SSB & DSB \\
\hline $\mathrm{a}$ & $1136.3 \pm 0.040$ & $49.656 \pm 0.019$ \\
$\mathrm{~b}$ & $-0.1735 \pm 0.0037$ & $0.0569 \pm 0.0020$ \\
$\mathrm{c}$ & $-0.9450 \pm 0.0034$ & $-0.9067 \pm 0.0054$
\end{tabular}

Table 1: Parameter fit for number of single resp. double strand breaks $\mathrm{Gy}^{-1}$ cell $^{-1}$ using a function of the form: $a+b x^{c}$. Energies are expressed in $\mathrm{MeV}$, and confidence levels from the fit procedure are given.

(iv) This repeated for all damage types and volumes with and without nano-particles.

(v) The ratio's of the different yields for these volumes provides the relative yield.

For reasons of simplicity we chose to concentrate on the yields of single and double strand breaks. The latter being defined as strand breaks on different DNA-helices not more than 10 base pairs apart[19].
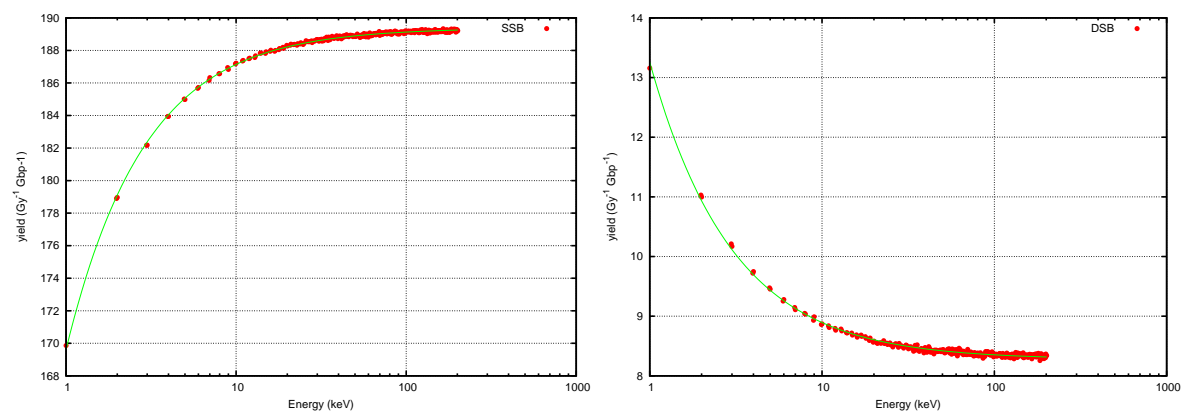

Figure 2: The yield of double strand breaks as defined by Nikjoo et al. fit with a function having the general form: $a+b x^{c}$, with $\mathrm{a}, \mathrm{b}$, and $\mathrm{c}$ being parameters.

\section{Results}

\subsection{Enhancement}

Figures $3 \mathrm{a}$ and $3 \mathrm{~b}$ show the maximal enhancements (i.e. $10 \%$ solution) of the dose in the volume containing GNP (target) compared to the skin and organ at risk structures. The skin ratio shows two local maxima at $60 \mathrm{keV}$ and at $90 \mathrm{keV}$. The OAR, which is positioned downstream of the target structure decreases monotonically reflecting a shielding effect due to the increased photon absorption in the target.

Figure 4 shows the absolute number of Auger or Coster-Krönig electrons (AE) generated in the target. This quantity depends on the atomic structure ( local maximum at $90 \mathrm{keV}$ ) and the depth of the target in the medium (local maximum at $40 \mathrm{keV}$ ). The highest number generated occurs at $90 \mathrm{keV}$. However, the energy of the $\mathrm{AE}$ in the latter case is much higher than those generated with lower energy. This can be seen in Figure 5 where the energy deposition of the various electrons is presented. Also note that in both cases the contribution of AE to the energy deposition process remains an order of magnitude lower. The largest part of the dose is deposited by photo-electric (PE) and knock-on electrons. 


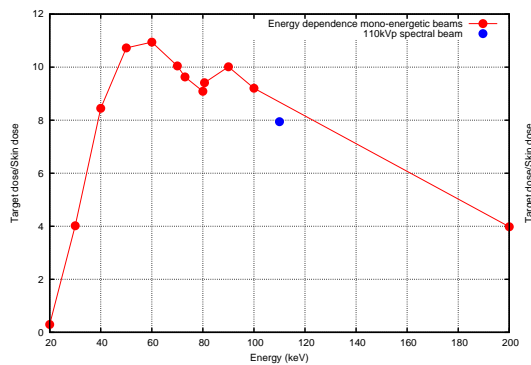

(a) Skin ratio

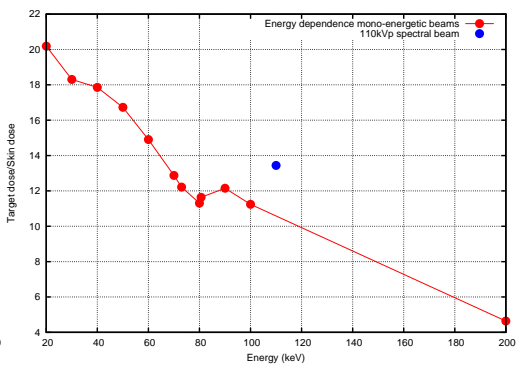

(b) OAR Enhancement

Figure 3: Enhancement ratio's as function of energy. For all points error bars are drawn but are too small for visualization, as all simulations were performed to yield errors smaller than $1 \%$

We plotted the absolute number of AE generated at the position of the GNP filled volume. Very low energy $\mathrm{X}$-rays are not able to reach this position. For this reason we see no expression of the photoactivation threshold for the M-shell AE.

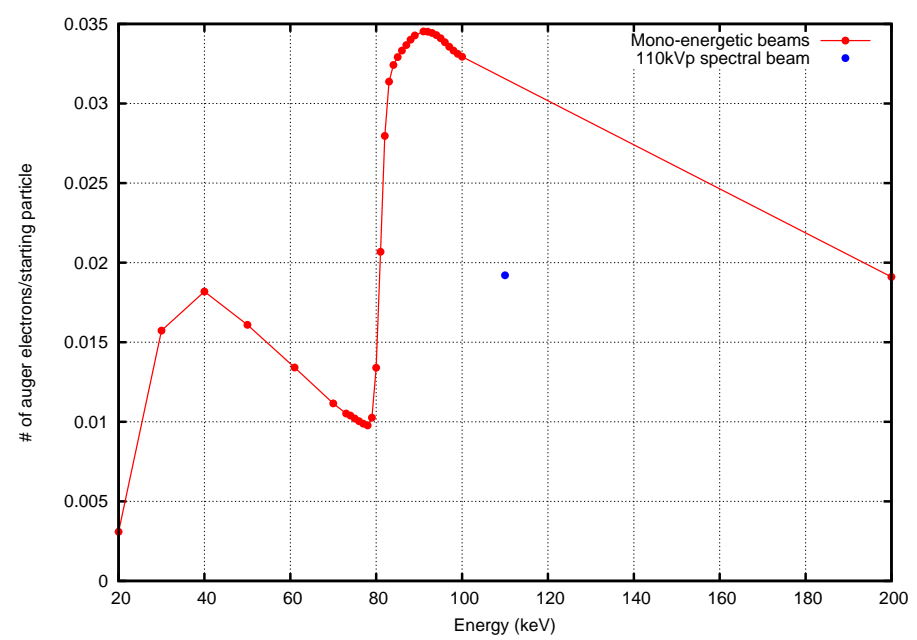

Figure 4: The number of electrons generated from the different possible channels in the medium containing the gold concentration as a function of energy for quasi mono-energetic beams. The point at $110 \mathrm{keV}$ represents the result from a bremsstrahlung-spectrum. The Auger electrons show a maximum around 40keV while an sudden increase is also noted at the $\mathrm{K}_{\alpha}$-edge. Note that there still is a substantial contribution of Auger electrons in the bremsstrahlung $(110 \mathrm{kVp})$ beam.

Figure 5 provides a visualization of the energy deposition by the different types of electrons at two different energies. It is the energy of each electron that deposits energy in the medium. For this reason we see a continuous contribution from the Auger electrons as they gradually lose their energy. In the $60 \mathrm{keV}$ beam only the Lshell electrons are generated at the $90 \mathrm{keV}$ beam the K-shell Auger electrons augment the dose. Auger electrons can be generated in different ways. The general mechanism is an atomic relaxation after ionization either from a photon interaction or from a direct electron interaction. Both are plotted separately in the plots. A very small 

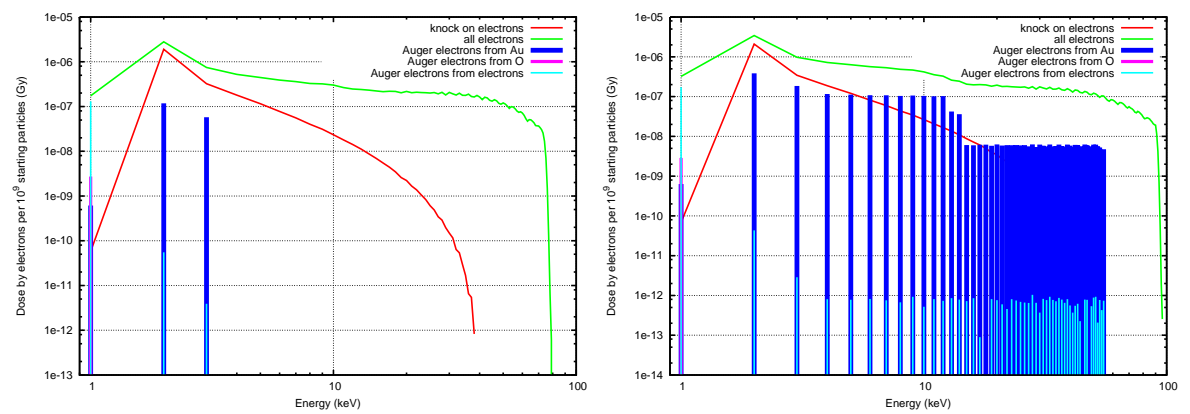

Figure 5: Energy spectra of energy depositing electrons in the activated volume, when using and energy below (left) the $\mathrm{K}_{\alpha}$-edge of golds, contrasted with the spectrum resulting from irradiation with an photon beam of $90 \mathrm{keV}$ which is above this edge (right).

contribution is noted from Auger electrons from oxygen. Both graphs extend slightly above their nominal energy as the spectra used where quasi-mono-chromatic and thus have a finite spectral width.

\subsection{Concentration}

Figure 6 shows the Absolute enhancement ratio as a function of the concentration of gold in the cell. $E_{a}$ was fit with a second order polynomial, where the quadratic factor is small with respect to the other factors. Showing that the dose enhancement is quasilinear in the sense that the quadratic term in the polynomial has a small contribution. When the second order polynomial is denoted as $f(x)=a x^{2}+b x+c$. Then the coefficients are: $\mathrm{a}=(-0.0195 \pm 0.0005), \mathrm{b}=(1.386 \pm 0.004)$, and $\mathrm{c}=(1.003 \pm 0.003)$. Fixing the $c$-coefficient to 1 gives comparable results. If a linear fit is performed the slope is given by $(1.26 \pm 0.02)$, a number which can be used to easily predict the impact of concentration changes.

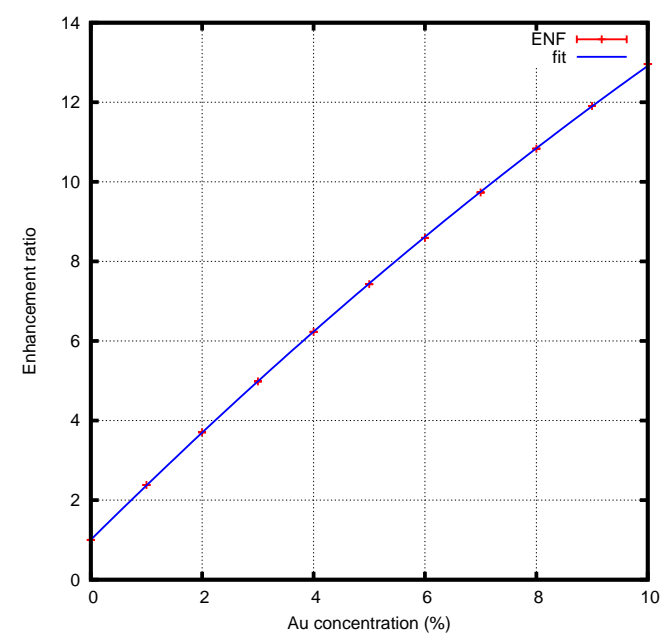

Figure 6: Graph depicting the variation of the absolute enhancement factor $\left(E_{a}\right)$ as a function of the gold concentration. A second order polynomial is fit to the values using a $\chi^{2}$-minimization. 


\subsection{Biological effect}

Figure 7 shows the yield per cell and per Gy of single strand breaks (SSB) and double strand breaks (DSB) as a function of energy. This for the three volumes under consideration. The target volume containing GNP reflects the atomic structure in the calculated yields. One can easily identify the when auger electrons are generated as a function of beam energy. The addition generates increases in DSB and lowers the contributions of SSB. However, the added tail of high energy electrons mainly affects the DNA through SSB's. It is only for very low energies $(10-20 \mathrm{keV})$ that the addition of GNP changes the ratio of SSB to DSB to give the dose deposition a more high LET character. For deeper lying structures there is no data available for $10 \mathrm{keV}$ as the photons do not penetrate deep enough to generate a meaningful contribution.
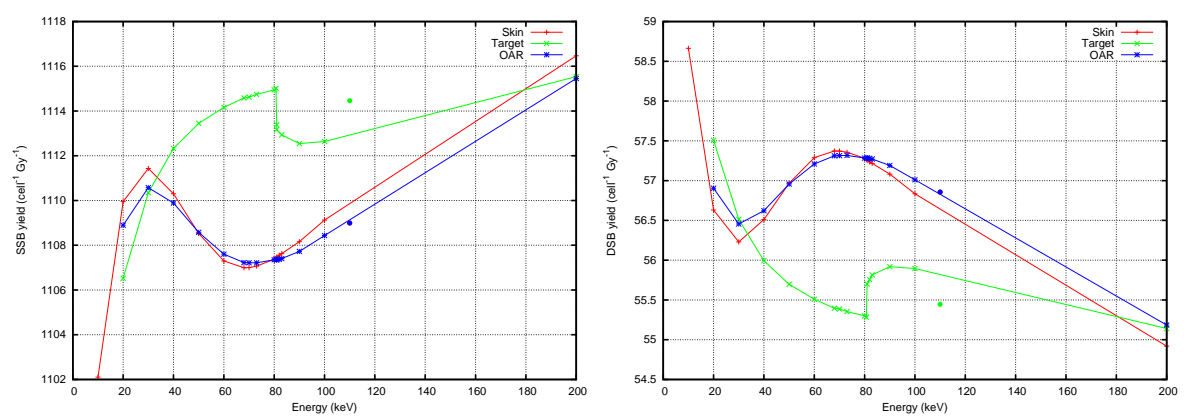

Figure 7: Plots for generation of single strand breaks (left) and double strand breaks (right) as a function of energy for a given dose. The curves are complementary as the number of SSB's goes down the DSB's go up. In the target volume the atomic structure and resonant levels generating auger electrons are visible but have a limited impact on the overall result with respect to the deposition being of a high or low LET nature.

The spectrum in the target does contain more low energy electrons compared to the OAR's. This advantage is negated by the additional tail of high energy electrons as illustrated in Fig. 8 where a cumulative representation of the dose deposition in double strand breaks is shown for a beam energy of $60 \mathrm{kev}$.

\section{Discussion}

From the data available here, one can conclude that there exists a necessity to perform full monte carlo simulation based for these type of treatments. Not only does the enhancement change with energy it is also imperative to monitor the concentration of the nano-particles during treatment. Before treatments can be started methodologies need to be developed to monitor concentrations adequately. The monitoring of the concentration and the error of this specific measurement has a direct impact on the dosimetry as we find a relationship of concentration to dose which is close to unity. This implies that the uncertainty of the dosimetric planning is at least equal to the uncertainty of this measurement.

The choice of energy of the radiation beam for treatment does not seem to be a critical issue, as long as radiation reaches the intended volume. So, it might not be necessary to build expensive high output mono-energetic photon sources. However, to determine the concentration it might be necessary to do so albeit with a lower 


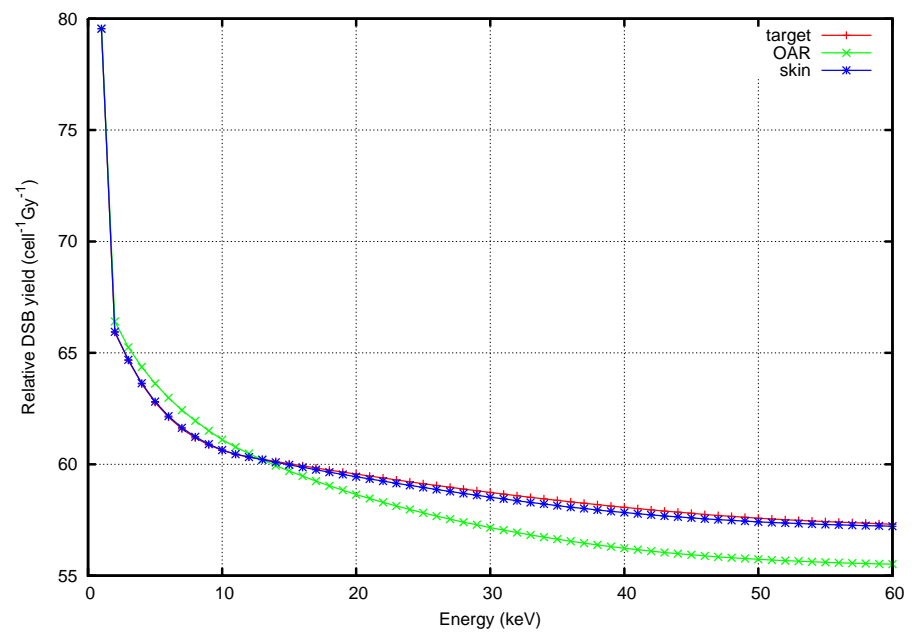

Figure 8: Cumulative graph of the contribution to dose from double strand breaks. For each energy bin the contribution is added to the higher energies. For the GNP volume the contribution of low energy auger electrons has a relatively high effect. As the higher energy electrons are added the effect diminishes.

photon flux [20, 21]. A possible alternative for measurement is the use of the same markers with nano-particles attached, but where the gold is replaced by a radioactive isotope[22]. Using methodology developed in nuclear medicine imaging techniques the concentration and its variation can then be monitored over time. This approach gives up some of the advantages as the radioactive particles will deliver a dose in the manner we are trying to avoid. The dose, however, is only for imaging purposes and is more limited than when a therapeutic procedure is attempted. A further approach could be to measure concentration changes using other nano-particles attached to same targeting agent and use non-ionizing techniques for visualization[23]. A drawback of this technique is that due to the use of different nano-particles, the uptake and concentration dynamics could be slightly different from the therapeutically enhanced targeting molecules.

The impact of Auger electrons seems to be limited as they only consist of a small fraction of the dose depositing electrons. Only at very low energies do they seem to have an effect in increasing the efficiency of the dose deposition. Using such low energies limits the use to superficial tumors or warrants the use of intra-operative techniques or brachytherapy techniques using low energy sources like ${ }^{125} \mathrm{I}$ or electronic brachytherapy sources[24].

The data presented here does not stand alone and can be compared to experimental data provided by other authors who have also looked at the possible optimal energies to use for nanoparticle enhanced treatment using in-vitro techniques as well as implementations through animal testing. Rahman and colleagues[25] used clinical superficial X-ray machines to irradiate cells containing GNP's at different concentrations. Nominal energies of 80 and $150 \mathrm{kVp}$ showed dose enhancement. In agreement with our calculations they show a dependence of cell survival on energy, whereby the $80 \mathrm{kVp}$ beam is shown to be more effective. It is not clear however what the exact spectrum is that was used. In theory it could be possible to predict the overall dose enhancement of a poly-chromatic beam from the weighted sum of 
mono-chromatic beams. Important to note is also that Rahman and colleagues found increased cytotoxicity depending on the concentration, a factor not taken into account in our analysis.

Brun et al. performed a comparable study also using different superficial energies characterized by the effective energy of the X-ray beam $(14.8,24.4,29.8,42.4$, 49, and $70.1 \mathrm{keV}$ ). The highest enhancement was noted at $49 \mathrm{keV}$, which could be considered as a $50 \mathrm{keV}$ beam. This again agrees with our calculations.

In the papers discussed above the dose is determined in water and the enhancement factors are determined by estimating increase in cell kill. In our approach we attempt to resolve the difference between physical dose and biological effect. Indeed figure 7 has the physical dose removed and shows that most of the enhancement proposed here is a consequence of increased photon absorption rather than an effect of short ranged auger electrons.

Finally, an interesting approach is presented by Biston et al., where cisPlatinum is introduced in rats with a glioma tumor[26]. The tumor is subsequently irradiated with quasi-mono-energetic $\mathrm{X}$-ray beams just below and above the $\mathrm{K}$-edge of platinum $(\mathrm{Z}=78)$. This group reports and increase of DSB damage at the higher energy. This is in agreement with figure 7 where we see an substantial increase just above the $\mathrm{K}$-edge. However, the group has not tested their hypothesis at other energies. From the same figure we see that there are other energies that provide a better DSB/SSB ratio. From figure 5 we see that in all cases, and also at the "photo-activation" energy, the contribution of auger electrons to the dose is an order of magnitude lower than contribution from other sources. Therefore, it might not be necessary to have mono-chromatic sources and that sources with a sufficiently high flux could be adequate. Proposals of converting conventional linear accelerators using low-Z targets come to mind as proposed by Robar et al, [27].

[1] T. K. Nayak, J. P. Norenberg, T. L. Anderson, E. R. Prossnitz, M. G. Stabin, R. W. Atcher, Somatostatin-receptor-targeted [alpha]-emitting 213Bi is therapeutically more effective than [beta]-emitting 177Lu in human pancreatic adenocarcinoma cells, Nuclear Medicine and Biology 34 (2) (2007) $185-193$.

[2] M. Pless, C. Waldherr, H. Maecke, C. Buitrago, R. Herrmann, J. Mueller-Brand, Targeted radiotherapy for small cell lung cancer using 90Yttrium-DOTATOC, an Yttrium-labelled somatostatin analogue: a pilot trial, Lung Cancer 45 (3) (2004) 365 - 371.

[3] M. Miederer, D. A. Scheinberg, M. R. McDevitt, Realizing the potential of the actinium225 radionuclide generator in targeted alpha particle therapy applications, Advanced Drug Delivery Reviews 60 (12) (2008) 1371 - 1382, delivery Systems for the Targeted Radiotherapy of Cancer.

[4] A. Otte, S. M. Weiner, M. Cybulla, Is radiation nephropathy caused by Yttrium-90?, Lancet 359 (9310) (2002) 979; author reply 979.

[5] T. Schumacher, C. Waldherr, J. Mueller-Brand, H. Maecke, Kidney failure after treatment with 90Y-DOTATOC, Eur J Nucl Med Mol Imaging 29 (3) (2002) 435.

[6] T. M. Behr, M. Behe, G. Kluge, M. Gotthardt, M. L. Schipper, S. Gratz, R. Arnold, W. Becker, D. M. Goldenberg, Nephrotoxicity versus anti-tumour efficacy in radiopeptide therapy: facts and myths about the Scylla and Charybdis, Eur J Nucl Med Mol Imaging 29 (2) (2002) $277-279$.

[7] M. Cybulla, S. M. Weiner, A. Otte, End-stage renal disease after treatment with 90YDOTATOC, Eur J Nucl Med 28 (10) (2001) 1552-1554.

[8] J. F. Hainfeld, D. N. Slatkin, H. M. Smilowitz, The use of gold nanoparticles to enhance radiotherapy in mice, Phys Med Biol 49 (18) (2004) N309-315.

[9] F. Verhaegen, B. Reniers, F. Deblois, S. Devic, J. Seuntjens, D. Hristov, Dosimetric and microdosimetric study of contrast-enhanced radiotherapy with kilovolt x-rays, Physics in Medicine and Biology 50 (15) (2005) 3555-3569.

[10] S. Cho, Estimation of tumour dose enhancement due to gold nanoparticles during typical radiation treatments: a preliminary Monte Carlo study, Phys Med Biol 50 (15) (2005) N163N173. 
[11] S. H. Cho, B. L. Jones, S. Krishnan, The dosimetric feasibility of gold nanoparticle-aided radiation therapy (GNRT) via brachytherapy using low-energy gamma-/x-ray sources, Phys Med Biol 54 (16) (2009) 4889-4905.

[12] J. D. Carter, N. N. Cheng, Y. Qu, G. D. Suarez, T. Guo, Nanoscale energy deposition by X-ray absorbing nanostructures, Journal of physical chemistry B 111 (40) (2007) 11622-11625.

[13] E. Silver, A. Pradhan, Y. Yu, Tu-d-352-08: Innovative instrumentation for resonant cancer theranostics, Med Phys 35 (6) (2008) 2899-2899. URL http://link.aip.org/link/?MPH/35/2899/3

[14] J. Chen, A compilation of microdosimetry for uniformly distributed Auger emitters used in medicine, Int J Radiat Biol 84 (12) (2008) 1027-1033.

[15] T. Urashima, H. Nagasawa, K. Wang, S. J. Adelstein, J. B. Little, A. I. Kassis, Induction of apoptosis in human tumor cells after exposure to Auger electrons: comparison with gammaray exposure, Nucl Med Biol 33 (8) (2006) 1055-1063.

[16] P. Balagurumoorthy, K. Chen, S. J. Adelstein, A. I. Kassis, Auger electron-induced doublestrand breaks depend on DNA topology, Radiat Res 170 (1) (2008) 70-82.

[17] L. S. Waters, G. W. McKinney, J. W. Durkee, M. L. Fensin, J. S. Hendricks, M. R. James, R. C. Johns, D. B. Pelowitz, The MCNPX Monte Carlo radiation transport code, in: Albrow, M and Raja, R (Ed.), Hadronic Shower Simulation Workshop, Vol. 896 of AIP conference proceedings, Amer Inst Physics, 2007, pp. 81-90, Hadronic Shower Simulation Workshop, Batavia, IL, SEP 06-08, 2006.

[18] V. Semenenko, R. Stewart, Fast Monte Carlo simulation of DNA damage formed by electrons and light ions, Phys Med Biol.

[19] H. Nikjoo, P. O'Neill, D. T. Goodhead, M. Terrissol, Computational modelling of low-energy electron-induced DNA damage by early physical and chemical events, Int J Radiat Biol 71 (5) (1997) 467-473.

[20] M. Gambaccini, A. Tuffanelli, A. Taibi, A. Del Guerra, Spatial resolution measurements in quasimonochromatic $\mathrm{x}$ rays with mosaic crystals for mammography application, Medical Physics 28 (4) (2001) 412-418.

[21] P. Baldelli, A. Taibi, A. Tuffanelli, M. Gilardoni, M. Gambaccini, A prototype of a quasimonochromatic system for mammography applications, Physics in Medicine and Biology 50 (10) (2005) 2225-2240.

[22] K. Roy, S. Lahiri, A green method for synthesis of radioactive gold nanoparticles, Green Chemistry 8 (12) (2006) 1063-1066.

[23] C. Sun, C. Fang, Z. Stephen, O. Veiseh, S. Hansen, D. Lee, R. G. Ellenbogen, J. Olson, M. Zhang, Tumor-targeted drug delivery and MRI contrast enhancement by chlorotoxin-conjugated iron oxide nanoparticles, Nanomedicine 3 (4) (2008) 495-505.

[24] M. J. Rivard, S. D. Davis, L. A. DeWerd, T. W. Rusch, S. Axelrod, Calculated and measured brachytherapy dosimetry parameters in water for the xoft axxent x-ray source: An electronic brachytherapy source, Medical Physics 33 (11) (2006) 4020-4032. URL http://link.aip.org/link/?MPH/33/4020/1

[25] W. N. Rahman, N. Bishara, T. Ackerly, C. F. He, P. Jackson, C. Wong, R. Davidson, M. Geso, Enhancement of radiation effects by gold nanoparticles for superficial radiation therapy, Nanomedicine 5 (2) (2009) 136-142.

[26] M. Biston, A. Joubert, J. Adam, H. Elleaume, S. Bohic, A. Charvet, F. Esteve, N. Foray, J. Balosso, Cure of fisher rats bearing radioresistant F98 glioma treated with cis-platinum and irradiated with monochromatic synchrotron X-rays, Cancer Research 64 (7) (2004) 23172323.

[27] J. L. Robar, Generation and modelling of megavoltage photon beams for contrast-enhanced radiation therapy, Phys. med. biol. 51 (21) (2006) 5487-5504. 